\title{
Stem cells from human exfoliated deciduous teeth ameliorate type II diabetic mellitus in Goto-Kakizaki rats
}

Nanquan Rao ${ }^{1 \dagger}$, Xiaotong Wang ${ }^{2 \dagger}$, Yue Zhai ${ }^{1}$, Jingzhi $\mathrm{Li}^{1}$, Jing Xie ${ }^{3}$, Yuming Zhao ${ }^{1 *}$ and Lihong Ge ${ }^{1 *}$

\begin{abstract}
Background: By 2030, diabetes mellitus (DM) will be the 7th leading cause of death worldwide. Type 2 DM (T2DM) is the most common type of DM and is characterized by insulin resistance and defective $\beta$-cell secretory function. Stem cells from human exfoliated deciduous teeth (SHED) are favorable seed cells for mesenchymal stem cells (MSCs)based therapy due to their higher proliferation rates and easier access to retrieval. Currently, no study has revealed the therapeutic efficiency of MSCs for T2DM in Goto-Kakizaki (GK) rats. Hence, we aimed to explore the effect of SHED on T2DM in GK rats.

Materials and methods: We investigated the effects of SHED on the progression of T2DM in GK rats. SHED and bone marrow mesenchymal stem cells (BMSCs) were injected via the tail vein. Body weight, fasting blood glucose and non-fasting blood glucose were measured before and after administration. At 8 weeks after injection, intraperitoneal insulin tolerance tests (IPITTs) and insulin release tests (IRTs) were performed. Additionally, hematoxylin-eosin (HE) staining, periodic acid-Schiff (PAS) staining and double-label immunofluorescence staining were used to explore the pathological changes in pancreatic islets and the liver. Immunohistochemistry (IHC) was employed to detect SHED engraftment in the liver. Additionally, real-time PCR and western blotting were used to explore glycogen synthesis, glycolysis and gluconeogenesis in the liver.
\end{abstract}

Results: At 8 weeks after SHED injection, T2DM was dramatically attenuated, including hyperglycemia, IPGTT and IRT. Additionally, histological analysis showed that SHED injection improved pancreatic islet and liver damage. Real-time PCR analysis showed that SHED significantly reversed the diabetic-induced increase of G-6-Pase, Pck1 and PK; and significantly reversed the diabetic-induced decrease of GSK3 $\beta$, GLUT2, and PFKL. In addition, western blotting demonstrated that SHED significantly reversed the diabetic-induced increase of G-6-Pase and reversed the diabetic-induced decrease of GLUT2, GSK3 $\beta$ and PFKM.

Conclusion: Stem cells from human exfoliated deciduous teeth offers a potentially effective therapeutic modality for ameliorating T2DM, including hyperglycemia, insulin resistance, pancreatic islets and liver damage, and decreased glycogen synthesis, inhibited glycolysis and increased gluconeogenesis in the liver.

Keywords: Stem cells from human exfoliated deciduous teeth (SHED), Type 2 diabetes mellitus, Goto-Kakizaki (GK) rats, Insulin resistance

\footnotetext{
*Correspondence: yumingzhao70@sina.com; gelihong0919@163.com

${ }^{\dagger}$ Nanquan Rao and Xiaotong Wang contributed equally to this work.

1 Department of Pediatric Dentistry, Peking University School

and Hospital of Stomatology, 22 Zhongguancun Avenue South, Haidian

District, Beijing 100081, People's Republic of China

Full list of author information is available at the end of the article

Nanquan Rao and Xiaotong Wang contributed equally to this work.
}

(c) The Author(s) 2019. This article is distributed under the terms of the Creative Commons Attribution 4.0 International License (http://creativecommons.org/licenses/by/4.0/), which permits unrestricted use, distribution, and reproduction in any medium, provided you give appropriate credit to the original author(s) and the source, provide a link to the Creative Commons license, and indicate if changes were made. The Creative Commons Public Domain Dedication waiver (http://creativecommons.org/ publicdomain/zero/1.0/) applies to the data made available in this article, unless otherwise stated. 


\section{Background}

Currently, though artificial synthesis and extensive application of insulin have substantially decreased the mortality associated with DM and improved the quality of life of DM patients and related complications, more than 400 million people throughout the world with DM continue to suffer from devastating secondary complications [1]. T2DM is the most common type of DM and is characterized by progressively inexorable $\beta$-cell dysfunction and insulin resistance in skeletal muscle, adipose tissue, and the liver [2]. In addition, currently available therapeutic regimens either target insulin resistance or insulin deficiency [3]. Excellent metabolic control without the need for exogenous insulin can be achieved with pancreas transplantation or pancreatic islet transplantation. While the procedure is associated with adverse effects in a limited number of available donors, immunosuppressive regimens [4] have immunological risks that affect longterm survival [5].

Therefore, MSCs appear to be an ideal tool for treating $\mathrm{DM}$ and the related secondary complications as the cells can be easily isolated from bone marrow, adipose tissue, cord blood and dental pulp and can be rapidly expanded in vitro [6]. Importantly, MSCs are hypoimmunogenic. When systemically administered, MSCs home to injured organs, contribute to tissue regeneration and have been transplanted into human patients with different diseases with beneficial effects and without major toxicity [7-9]. The antidiabetic effect of autologous and allogeneic MSCs has been demonstrated in different animal models of and patients with T1DM [10-12] and T2DM [13, 14]. It is widely accepted that MSCs might contribute to tissue regeneration due to their ability to regulate the local microenvironment by paracrine mechanisms [15-17]. MSCs limit the expansion and cytotoxic activity of T lymphocytes and stimulate the appearance of regulatory $\mathrm{T}$ cells [18]. Furthermore, MSCs secrete anti-inflammatory cytokines and inhibit the expression of pro-inflammatory cytokines by immune cells $[19,20]$. Moreover, MSCs are able to produce both in vitro and in vivo anti-apoptotic and mitogenic factors, including epidermal growth factor (EGF), hepatocyte growth factor (HGF), insulin-like growth factor-1 (IGF1), and basic fibroblast growth factor (bFGF). The biological effects of these trophic factors can be direct (triggering intracellular signaling) or indirect (inducing neighboring cells to secrete other bioactive factors) [21, 22].

Miura et al. [23] found that SHED have superior proliferative capacity, self-renewal ability, and multidirectional differentiation potential. SHED are MSCs derived from dental pulps in exfoliated deciduous teeth from young patients. In addition, SHED have immunomodulatory abilities [24]. SHED have a good prospect in the treatment of T1DM [25], liver fibrosis [26], lupus erythematosus [27], and spinal cord injury [28].

Therefore, we hypothesize that SHED could be beneficial for the progression of T2DM. In this study, GK rats, a non-obese and spontaneous (genetic) T2DM model, was first used to examine the effect of MSCs on T2DM.

\section{Materials and methods}

\section{SHED isolation, in vitro expansion and characterization}

The SHED donors were patients aged 6 to 8 years old from a pediatric clinic. The protocol was approved by the Ethics Committee (PKUSSIRB-201630091). The isolation of SHED was performed following Miura et al's reports [22]. Briefly, the pulp tissues were collected from deciduous teeth, cut into small pieces, then digested with $0.1 \%$ type I collagenase (Sigma-Aldrich, St. Louis, MO, USA) and dispase (Sigma-Aldrich, St. Louis, MO, USA) in $\alpha$-MEM (Hyclone, Logan, UT, USA) at $37^{\circ} \mathrm{C}$ for $60 \mathrm{~min}$. Then, $\alpha$-MEM containing 20\% FBS (Gibco, Mulgrave, VIC, Australia) was added and subsequently centrifuged at $1000 \mathrm{rpm} / \mathrm{min}$ for $5 \mathrm{~min}$. Next, the supernatant was discarded and the cell pellet was resuspended and cultured at room temperature in $\alpha$-MEM containing $20 \%$ FBS, $100 \mathrm{U} / \mathrm{mL}$ penicillin and $100 \mu \mathrm{g} / \mathrm{mL}$ streptomycin (Solarbio, Beijing, China) at $37{ }^{\circ} \mathrm{C}$ in $5 \% \mathrm{CO}_{2}$ for 3 days. The culture solution was changed every 3 or 4 days.

Human BMSCs were purchased from ScienCell (San Diego, California, USA) and cultured in $\alpha$-MEM containing $10 \% \mathrm{FBS}, 100 \mathrm{U} / \mathrm{mL}$ penicillin and $100 \mu \mathrm{g} / \mathrm{mL}$ streptomycin.

When monolayer SHED and BMSCs confluence was observed, the cells were passaged at a ratio of 1:3 and cultured with $\alpha$-MEM supplemented with $10 \%$ FBS and $1 \%$ antibiotics at $37{ }^{\circ} \mathrm{C}$ in $5 \% \mathrm{CO}_{2}$. Cells from passage 3 to 4 were used in the present study.

The surface marker profiles of SHED were tested with flow cytometry. The third passage of cells was resuspended in cold PBS containing $2 \% \mathrm{FBS}$ at a concentration of $1 \times 10^{6}$ cells $/ \mathrm{mL}$ prior to adding the following monoclonal antibodies: CD34-PE, CD45-PE, CD73-PE, CD90FITC, CD105-FITC and CD146-PE (Beckman Coulter, Brea, CA, USA). The unmarked cells were used as a negative control. Finally, the stained cells were analyzed using a Beckman Coulter flow cytometry system (FC500, Beckman Coulter, Brea, CA, USA).

\section{Adipogenic and osteogenic differentiation}

To induce adipogenic differentiation, confluent adherent cells were cultured in $\alpha$-MEM containing $10 \% \mathrm{FBS}$, $100 \mathrm{U} / \mathrm{mL}$ penicillin and $100 \mu \mathrm{g} / \mathrm{mL}$ streptomycin, supplemented with $1 \mu \mathrm{M}$ dexamethasone (Sigma-Aldrich, St Louis, MO, USA), $100 \mu \mathrm{g} / \mathrm{mL}$ 3-isobutyl-1-methyxathines (Sigma-Aldrich, St Louis, MO, USA), $100 \mu \mathrm{M}$ 
indomethacin (Sigma-Aldrich, St Louis, MO, USA) and $200 \mu \mathrm{M} / \mathrm{mL}$ insulin (Sigma-Aldrich, St Louis, MO, USA), replaced every 2 days. After 21 days of stimulation, cell differentiation into lipid-laden adipocytes was confirmed by Oil Red O (Sigma-Aldrich, St. Louis, MO, USA) staining.

To induce osteogenic differentiation, confluent adherent cells were cultured in media containing 10\% FBS and $100 \mathrm{U} / \mathrm{mL}$ penicillin and $100 \mu \mathrm{g} / \mathrm{mL}$ streptomycin, supplemented with $0.1 \mu \mathrm{M}$ dexamethasone, $10 \mathrm{mM}$ $\beta$-glycerophosphate (Sigma-Aldrich, St. Louis, MO, USA) and $50 \mu \mathrm{g} / \mathrm{mL}$ ascorbate 2-phosphate (Sigma-Aldrich, St. Louis, MO, USA), replaced every 2 days. After 14 days of stimulation, cells that differentiated into hydroxyapatiteproducing osteoblasts were confirmed by Alizarin Red (Sigma-Aldrich, St. Louis, MO, USA) staining.

\section{Animal model and groups}

Twenty-five SPF grade GK rats (12 weeks old, male) were purchased from Changzhou Cavens Laboratory Animal Co., Ltd (Changzhou, Jiangsu, China). Eight SPF grade Wistar rats (12 weeks old, male) were purchased from Beijing Vital River Laboratory Animal Technology Co., Ltd (Beijing, China) as normal controls. The rats were maintained on a $12 \mathrm{~h}$ light: $12 \mathrm{~h}$ dark cycle with free access to rodent chow and water. All animal experiments were conducted in accordance with the protocols and guidelines of the PU IRB Laboratory Animal Welfare Committee.

After GK rats consumed a high fat diet for 4 weeks, rats with a non-fasting blood glucose $\geq 11.1 \mathrm{mM}$ for 3 consecutive days were classified as T2DM rats. T2DM rats were randomly divided into three groups and eight normal Wistar rats were used as a normal control group. Then, T2DM rats were subsequently fed conventional chow. Rats in four groups received the following treatment: (1) normal Wistar rats without treatment (normal group, $\mathrm{n}=8$ ); (2) GK rats received SHED infusion (SHED group, $\mathrm{n}=8$ ); (3) GK rats received $1 \mathrm{~mL}$ of PBS infusion (PBS group, $n=8$ ); (4) GK rats received BMSC infusion (BMSCs group, $\mathrm{n}=8$ ). All rats in the four groups were observed for 8 weeks after administration.

In total, $4 \times 10^{6}$ cells, including SHED and BMSCs, were resuspended in $1 \mathrm{~mL}$ PBS and administered via tail vein to each rat. The PBS group received $1 \mathrm{~mL}$ PBS.

\section{Physical and biochemical assessment}

Body weight, fasting blood glucose and non-fasting blood glucose were measured each week.

At 8 weeks after administration, IPITTs and IRTs were performed. Plasma glucose levels were monitored throughout the experiments with ACCU-CHEK Performa (Roche Diagnostic, Basel, Switzerland). Serum insulin measurement was performed by ELISA (Rat Insulin Elisa Kit; Millipore, St. Charles, MO).

Fasting blood glucose (FBG) and fasting serum insulin (FINS) concentrations were measured in blood collected through tail prick at 8 weeks after MSCs injection. The homeostatic model assessment (HOMA) described previously was used to assess changes in pancreatic $\beta$-cell function (HOMA- $\beta$ ) and insulin resistance (HOMAIR) in treated groups during the experimental period (22). The following equations were used to calculate the HOMA-IR index and HOMA- $\beta$ index: HOMAIR index $=($ FBG $[$ in $\mathrm{mmol} / \mathrm{L}] 3$ FINS $[$ in units/L] $) / 22.5$ and HOMA-b $=\left(20^{*}\right.$ FINS $[$ in units $\left./ \mathrm{L}]\right) /(\mathrm{FBG} \quad$ in $\mathrm{mmol} / \mathrm{L}]-3.5)$.

\section{Pancreatic islet histological and immunohistochemical analysis}

After IPGTT tests, the rats were given free access to rodent chow and water. Three days later, the rats were sacrificed using $10 \%$ chloral hydrate (Solarbio, Beijing, China). Liver tissues and pancreatic tissue were collected for further investigation.

Pancreatic tissue was embedded in paraffin. Paraffin sections $(4 \mu \mathrm{m})$ were stained with hematoxylin-eosin reagent. The number of regular and irregular pancreatic islets was counted, and the ratio of irregular islets to total islets was calculated. Five slices from each rat were randomly collected for statistics.

For double-label immunofluorescence staining, sections were deparaffinized, rehydrated and submitted to Tris-EDTA, pH 9.0 antigen retrieval solution (Zhongshan Golden Bridge Biotechnology, Beijing, China) in a microwave for $30 \mathrm{~min}$. Next, $3 \%$ hydrogen peroxide (Zhongshan Golden Bridge Biotechnology, Beijing, China) was used to block the endogenous peroxide. Then, rabbit anti-rat insulin and mouse anti-rat glucagon primary antibodies (Abcam, Cambridge, UK) were added, and the specimens were incubated at $4{ }^{\circ} \mathrm{C}$ overnight and washed thoroughly with PBS. The sections were incubated with a secondary antibody (Alexa Fluor 488-conjugated goat anti-rabbit IgG and Alexa Fluor 594-conjugated goat anti-mouse IgG, Abcam, Cambridge, UK) at room temperature for $2 \mathrm{~h}$.

Subsequently, pancreatic sections were stained with DAPI (Solarbio, Beijing, China) and mounted. The sections were observed by fluorescence microscopy. $\alpha$-Cells and $\beta$-cells were recorded in each section, and the ratio of $\beta$-cells to total islet cells was calculated. Five slices from each rat were randomly collected for statistics.

Liver histological analysis and SHED detection in the liver Liver tissue was embedded in paraffin. Liver sections $(4 \mu \mathrm{m})$ were stained with PAS reagent (Solarbio, Beijing, 
China) to assess glycogen. Slices were analyzed under light microscopy, and images were captured with a digital camera.

Additionally, SHED engraftment in liver tissue was detected by immunohistochemistry in prepared sections as follows. Briefly, the sections were deparaffinized, rehydrated and submitted to Tris-EDTA, pH 9.0 antigen retrieval solution (Zhongshan Golden Bridge Biotechnology, Beijing, China) in a microwave for $30 \mathrm{~min}$. Next, $3 \%$ hydrogen peroxide (Zhongshan Golden Bridge Biotechnology, Beijing, China) was used to block the endogenous peroxide. Then, the sections were incubated with human DNA PKcs primary antibody (Proteintech, Chicago, IL, USA) at $4{ }^{\circ} \mathrm{C}$ overnight. After a thorough rinse with PBS, sections were incubated with a secondary antibody using a horseradish peroxidase polymer system (Zhongshan Golden Bridge Biotechnology, Beijing, China) at room temperature for $30 \mathrm{~min}$. The detection step was performed with diaminobenzidine (Zhongshan Golden Bridge Biotechnology, Beijing, China). Finally, sections were counterstained with hematoxylin to distinguish cell nuclei.

\section{Quantitative real-time PCR analysis and western blotting of the liver}

Total RNA was isolated from liver tissues using TRIzol reagent (Invitrogen, Carlsbad, CA, USA). Then, mRNA was reverse transcribed to cDNA by using a transcriptor first-strand cDNA synthesis kit (Takara Biotechnology, Dalian, Liaoning, China). RNA expression was assessed by real time reverse transcriptase polymerase chain reaction (PCR) using a SYBR Green System (7300 Real time System, Applied Biosystem, Carlsbad, CA, USA) according to the manufacturer's protocol. cDNA was amplified by PCR using the following primers (Table 1 ).

Liver samples were lysed in ice-cold RIPA buffer (Solarbio, Beijing, China) with protease inhibitor cocktail (Solarbio, Beijing, China). Protein quantification was performed by BCA assay (Cwbio, Beijing, China), and equal amounts of protein lysate $(50 \mu \mathrm{g})$ were separated by
10\% SDS-PAGE. Transfer to nitrocellulose membranes was performed in transfer buffer (Millipore, Shanghai, China). After, the membranes were blocked for $2 \mathrm{~h}$ in TBST buffer with 5\% BSA and probed with the PFKM, GSK3 $\beta$, GLUT2 (Proteintech, Chicago, IL, USA), G-6Pase (Abcam, Cambridge, UK), and $\beta$-actin antibodies (Proteintech, Chicago, IL, USA) overnight at $4{ }^{\circ} \mathrm{C}$. The membranes were washed with TBST buffer three times, followed by incubation with the appropriate HRP conjugated secondary antibody (Proteintech, Chicago, Il, USA) for $1 \mathrm{~h}$ at room temperature. Finally, the protein expression levels were detected by ECL (Solarbio, Beijing, China).

\section{Statistical analysis}

All data are presented as the mean \pm standard deviation (SD). Statistical analysis was performed using SPSS software (version 17.0; SPSS Inc., Chicago, IL, USA). Student's $t$ test was used to compare variables before and after administration. For multiple comparisons, one-way ANOVA analysis of variance was applied. Statistical significance was set at $\mathrm{P}<0.05$.

\section{Results}

\section{MSC culture and identification}

Stem cells from human exfoliated deciduous teeth and BMSCs exhibited typical fibroblast-like morphology. SHED were differentiated into osteogenic and adipogenic cells. In addition, SHED were identified by the surface markers CD73(+), CD90(+), CD105(+), CD146(+), CD34(-) and CD45(-) by flow cytometry (Fig. 1).

\section{Effects of SHED on physical and biochemical parameters}

Twenty-four of $25 \mathrm{GK}$ rats met the condition of non-fasting blood glucose $\geq 11.1 \mathrm{mM}$ for 3 consecutive days.

The weight of the rats in each group increased slowly. The body weight of the SHED group, BMSCs group and PBS group was significantly lower than that of the normal group during treatment. Within 8 weeks, fasting blood glucose and non-fasting blood glucose levels in the PBS

Table 1 Primers used for PCR amplifications

\begin{tabular}{|c|c|c|}
\hline & Forward primer & Reverse primer \\
\hline GADPH & 5'-TGGAGTCTACTGGCGTCTT-3' & 5'-TGTCATATTTCTCGTGGTTCA-3' \\
\hline G6Pase & 5'-AGGTGGTGGCTGGAGTCTTG-3' & 5'-CTGGAGGCTGGCATTGTAGATG-3' \\
\hline GLUT2 & $5^{\prime}-\mathrm{CCAGCACATACGACACCAGAC-3^{ \prime }}$ & $5^{\prime}-A C A C A G A C A G A G A C C A G A G C A T A-3^{\prime}$ \\
\hline GSK3 $\beta$ & 5'-CGAACTCCACCAGAGGCAATC-3 & 5'-CGAACTCCACCAGAGGCAATC-3' \\
\hline PK & 5'-AACCAATATGCCTGCCTTCCAA-3' & 5'-ACCAACTGCCACCTTCCACTA-3' \\
\hline PFKL & $5^{\prime}-C C A G C C A C C A T C A G C A A C A A-3^{\prime}$ & 5'-TGTCTGTCTTCATCTTCTCTGTCAT-3' \\
\hline Pck1 & 5'-AACTGTTGGCTGGCTCTCACT -3' & 5'-GGAACCTGGCGTTGAATGCTT-3' \\
\hline
\end{tabular}




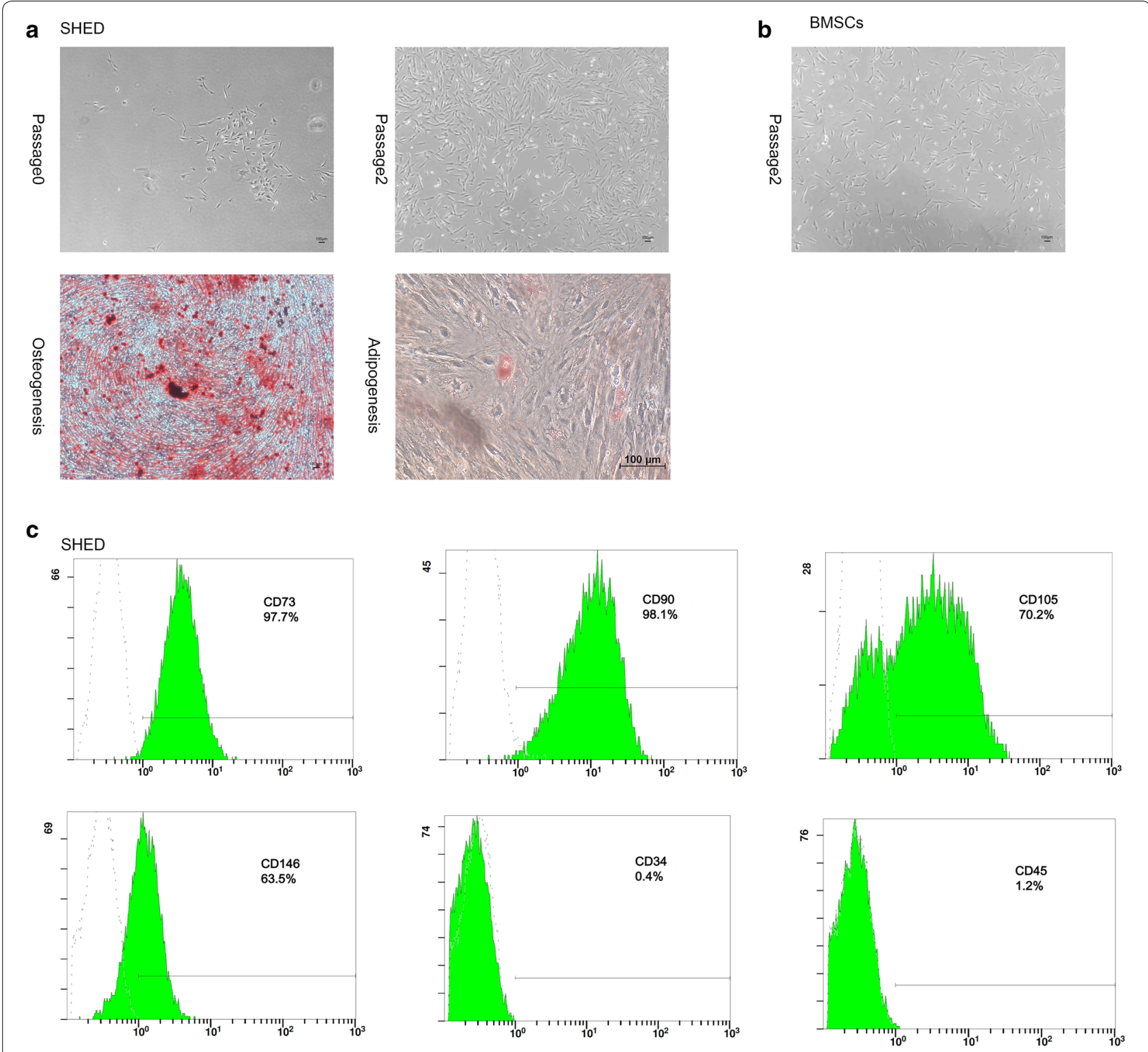

Fig. 1 Characterization of SHED and BMSCs. a Characterization of SHED. SHED showed a fibroblast-like morphology. Multilineage differentiation potency including osteogenesis as identified by Alizarin Red staining; adipogenesis as identified by Oil Red O staining. b BMSCs showed a fibroblast-like morphology. c Flow cytometry of SHED. SHED expressed low levels of CD34 (0.4\%) and CD45 (1.2\%) and expressed high levels of CD73 (97.7\%), CD90 (98.1\%), CD105 (70.2\%), and CD146 (63.5\%)

group, BMSCs group, and SHED group were significantly higher than those in the normal group. At 1 week and 8 weeks after injection, there was no statistically significant difference in fasting glucose and non-fasting glucose among the three groups of GK rats.

At 3 weeks after injection, fasting blood glucose in the SHED group was significantly lower than in the PBS group. At 5 weeks after injection, fasting blood glucose in the SHED group and BMSCs group were significantly lower than those in the PBS group. There was not a remarkable difference between the SHED group and BMSCs group.

Meanwhile, in the PBS group, there was no significant difference in fasting blood glucose after injection compared to before; in the BMSCs group, the fasting blood glucose level decreased remarkably, lasting 6 weeks; in the SHED group, fasting blood glucose was significantly lower after injection over 8 weeks except at 5 weeks after injection. 
Additionally, non-fasting blood glucose levels of T2DM rats were all significantly higher than those in the normal group during treatment. At 2, 3, 4, 6, and 7 weeks after injection, the non-fasting blood glucose level in the SHED group was significantly lower than that in the PBS group. There was no significant difference between the BMSCs group and PBS group, and there was no significant difference between the BMSCs group and SHED group. At 5 weeks after injection, non-fasting blood glucose levels in the SHED group and BMSCs group were significantly lower than in the PBS group, and there was no significant difference between the SHED group and BMSCs group.

The IPGTT test showed that blood glucose in the SHED group was significantly lower than in the PBS group at $1 \mathrm{~h}$ and $2 \mathrm{~h}$ after glucose injection.

Furthermore, HOMA- $\beta$ in the PBS group was significantly lower than in the normal group, and SHED and BMSCs injection reversed the decrease of HOMA- $\beta$ in GK rats; HOMA-IR in the SHED group and BMSCs group was significantly higher than in the PBS group, and there was no significant difference between the SHED group and BMSCs group (Fig. 2).

\section{Effects of SHED on pancreatic islet histological changes}

In the PBS group, obvious atrophy and morphologically irregular islets were observed. After calculation and statistics, the proportion of irregular islets in the SHED group, BMSCs group and PBS group was significantly higher than in the normal group, and the ratio of irregular islets in the SHED group and BMSCs group was significantly lower than the PBS group.

Immunofluorescence co-staining showed that the islets of normal rats had a round or elliptical shape, and islet $\beta$-cells were located in the center of the islets, which constituted most of the islets, and a small amount of scattered $\alpha$-cells were distributed on the outer edges of the islets. In the PBS group, the islet structure collapsed and was disordered, and $\alpha$-cells were not only located at the edge of the islets but also in the center of the islets, and the proportion of $\beta$-cells significantly decreased. Additionally, SHED and BMSCs injection significantly improved the abnormalities of the pancreatic islets (Fig. 3).

\section{Effects of SHED on liver histology and SHED detection in the liver}

Periodic acid-Schiff staining showed the storage of glycogen in the liver. In the normal group, glycogen is a

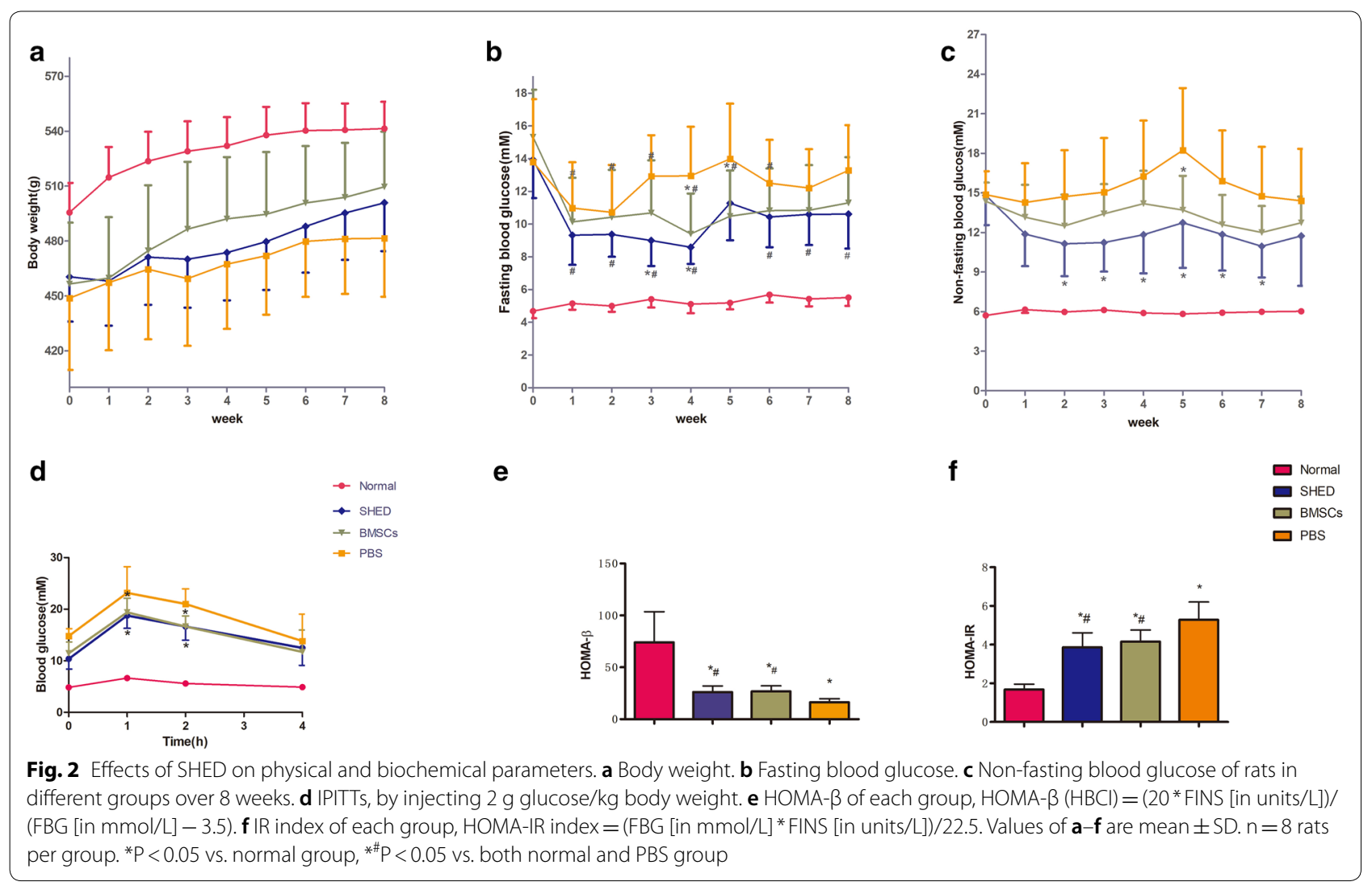




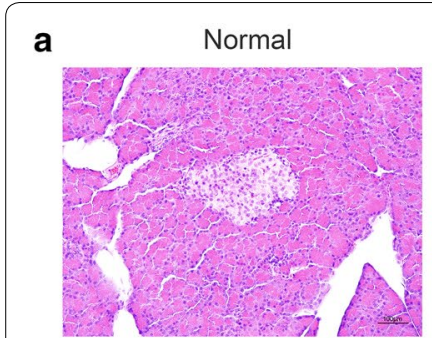

b
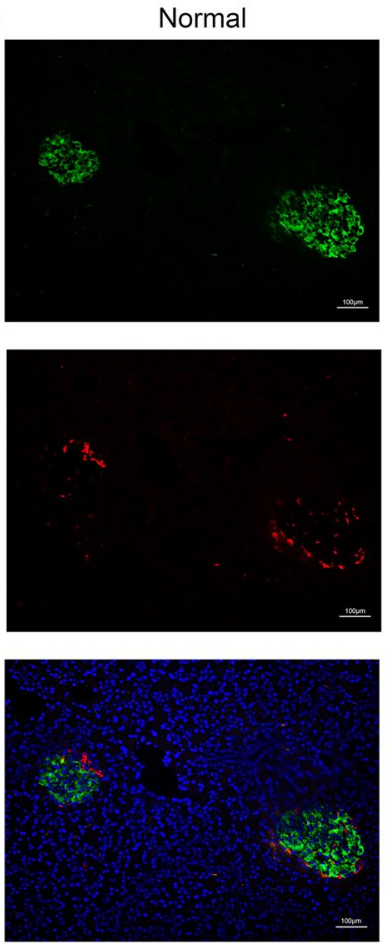

C

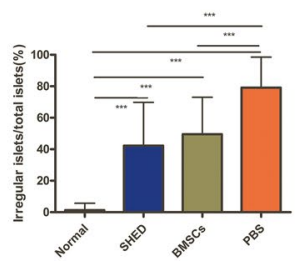

SHED

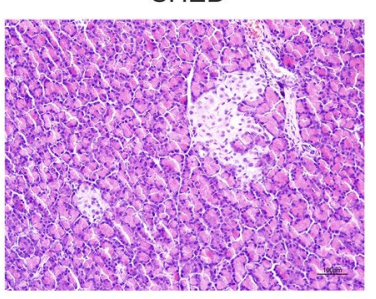

SHED
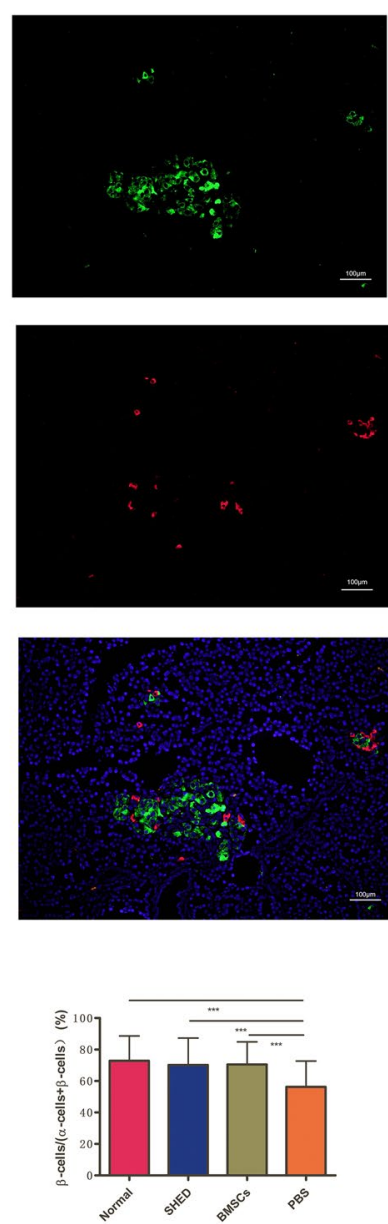

PBS
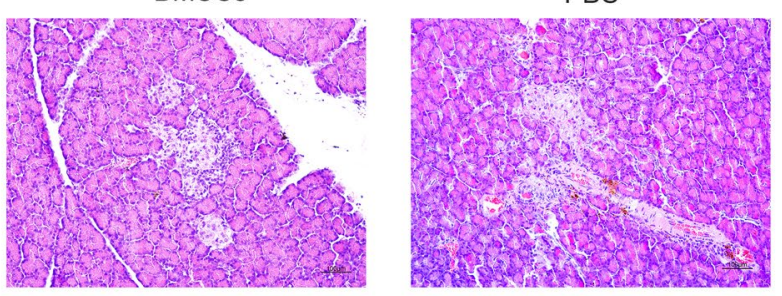

BMSCs
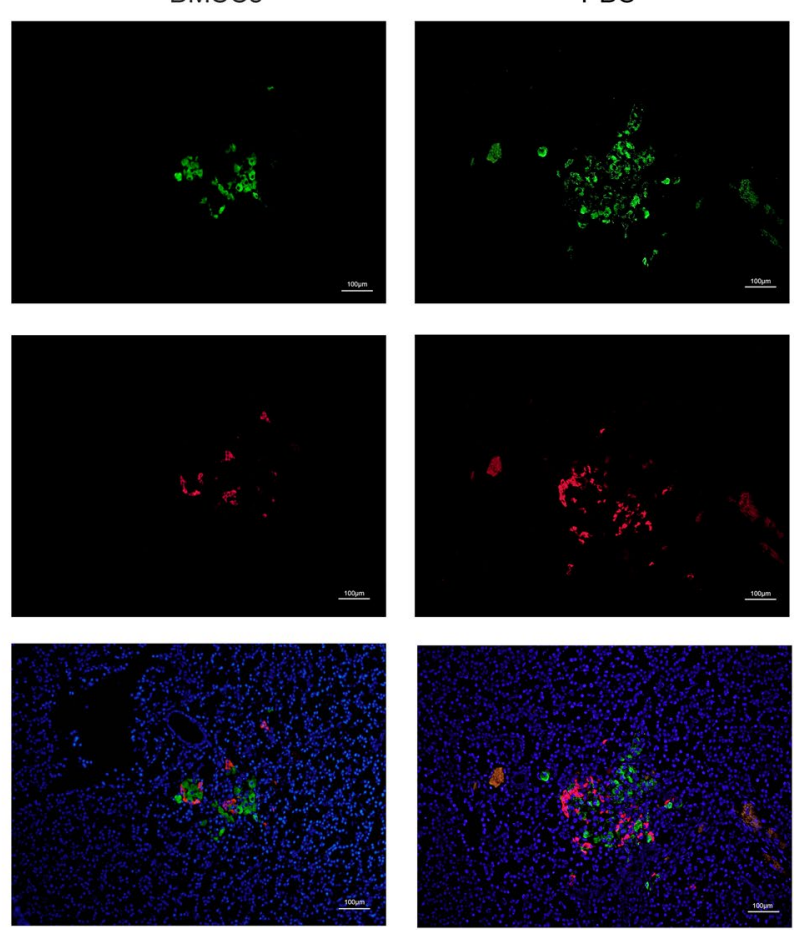

Fig. 3 Infusion of SHED promotes restoration of pancreatic islets in T2DM rats. a Pancreas histology was studied via HE staining, observed under light microscopy and focused on islet structures. $\mathbf{b}$ Pancreatic islets were characterized by immunofluorescence according to the presence and distribution of insulin-producing (green) and glucagon-producing (red) cells in four groups of rats at 8 weeks after infusion. Images were composite overlays of the individually stained nuclei, insulin and glucagon. SHED and BMSCs injection significantly improved abnormalities in pancreatic islets. c The proportion of the irregular islets and the proportion of $\beta$-cells in 4 group. ${ }^{* *} \mathrm{P}<0.001$

fine red particulate material that is widely present in the cytosol. In the PBS group, the amount of glycogen deposition was reduced and mottled vacuoles were observed while SHED and BMSC injection significantly improved the glycogen storage in T2DM rats.

Moreover, human DNA PKcs were detected in the liver at 8 weeks after MSC administration. SHED and BMSCs were found in liver tissue (Fig. 4). 

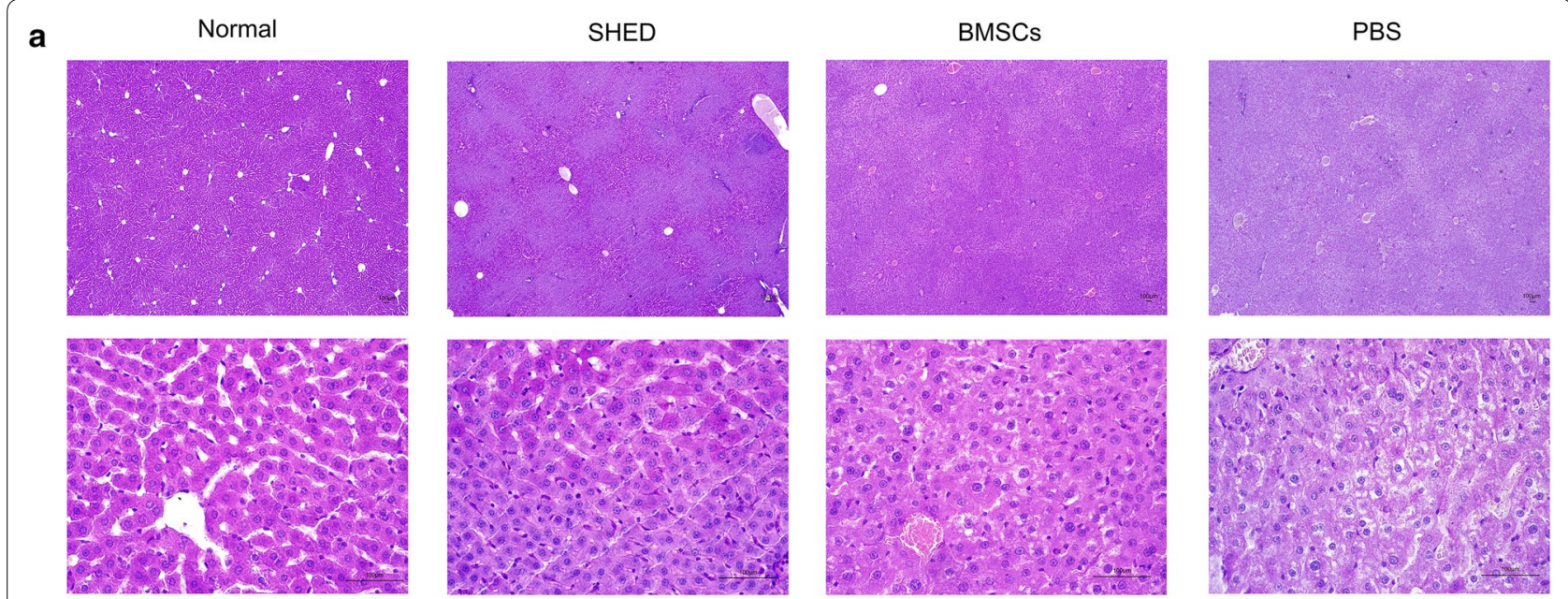

b

\section{SHED}

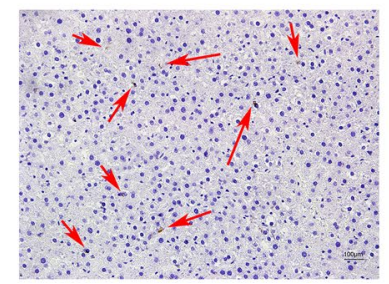

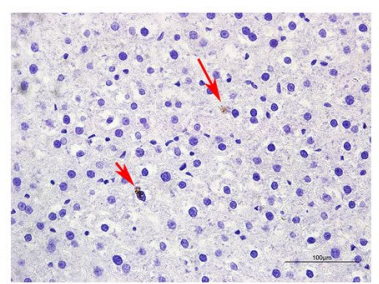
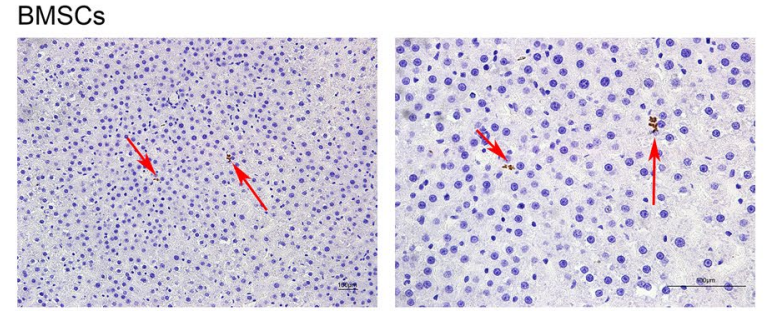

Fig. 4 Effects of SHED on liver histology and SHED engraftment in the liver. a PAS staining showed the storage of glycogen in the liver. SHED and BMSC injection significantly improved glycogen storage in T2DM. b Immunohistochemical representative micrograph of liver tissue from the SHED group and BMSCs group. Red arrow indicated SHED and BMSCs engraftment

\section{Quantitative real-time PCR analysis and western blotting of the liver}

Real-time PCR analysis showed that SHED and BMSCs significantly reversed the diabetic-induced increase of G-6-Pase, Pck1 and PK. In contrast, MSC injection significantly reversed the diabetic-induced decrease of GSK3b, GLUT2, and PFKL.

In addition, western blotting demonstrated that SHED and BMSCs significantly reversed the diabetic-induced increase of G-6-Pase and PK; in contrast, MSCs injection significantly reversed the diabetic-induced decrease of GSK3 $\beta$, GLUT2 and PFKM (Fig. 5).

\section{Discussion}

By 2030, diabetes mellitus (DM) will be the 7th leading cause of death worldwide [1]. The World Health Organization (WHO) claims that the incidence of DM has increased to 422 million individuals [2]. DM is associated with complications that affect patient quality of life. For example, more than $50 \%$ of diabetic patients present other physiological disorders, such as cardiovascular diseases (heart attacks and strokes), higher susceptibility to infections, kidney failure, and retinopathy. T2DM is the most common type of DM, and it is characterized by insulin resistance in skeletal muscle, adipose tissue, and the liver. Defective $\beta$-cell secretory function, fasting hyperglycemia and hyperinsulinemia, and increased hepatic glucose production are also typical characteristics of T2DM [3-5].

The antidiabetic effect of MSCs, such as BMSCs, umbilical cord blood (UCB) MSCs, and adipose tissuederived MSCs (ADSCs), has been demonstrated in different animal models and patients with T1DM [10-12] and T2DM [13, 14]. Transplantation of syngeneic or allogeneic MSCs is useful in preventing diabetes onset and also retarding its progression.

In our research, the present data clearly demonstrated that SHED administration ameliorated T2DM in GK rats, including hyperglycemia, the function and structure of pancreatic islets and insulin resistance in the liver. In addition, we found that there was no significant difference of the effects on DM between SHED and BMSCs.

Of note, at 3 weeks after injection, fasting blood glucose in the SHED group was significantly lower than in the PBS group. In the SHED group, fasting blood glucose was significantly lower after injection over 8 weeks except at 5 weeks. At 2, 3, 4, 6, and 7 weeks after injection, the non-fasting blood glucose level in the SHED 


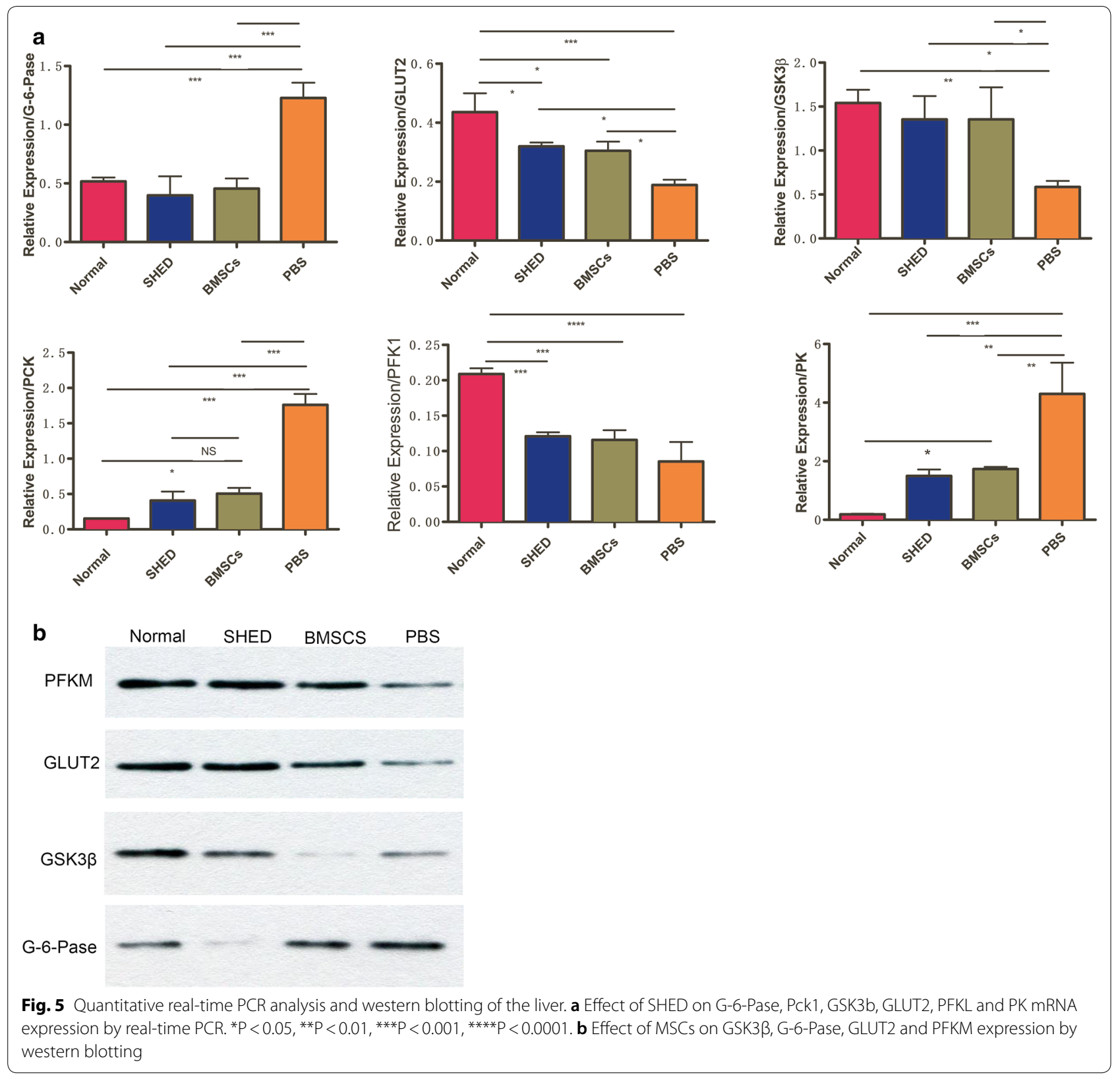

group was significantly lower than in the PBS group. At 5 weeks after administration, non-fasting blood glucose levels in the BMSCs and SHED groups were significantly lower than in the PBS group, and there was no significant difference between the BMSCs group and the SHED group. Our results differ from that of previous studies, which showed that the non-fasting blood glucose level decreased significantly during the entire treatment. One possible reason was that the animal model in this research was different from STZ-induced diabetic animals in the previous studies. This study is the first time that GK rats were used to explore the effect of MSC on
T2DM. GK rats, a non-obese and spontaneous (genetic) T2DM experimental model, have been widely used to investigate the development of T2DM and its complications. These animals were obtained by repeated selective breeding of glucose intolerant Wistar rats exhibiting peripheral insulin resistance and impaired insulin secretion [29, 30]. Another reason may be the differences among individual GK rats, so a study with a large sample is necessary in the future.

Regarding the effect of SHED on fasting glucose and non-fasting glucose, we supposed that SHED may influence blood glucose levels by decreasing insulin resistance 
in T2DM rats, so IPGTT tests were performed and the HOMA- $\beta$ index and HOMA-IR index were calculated. The results suggested that SHED can ameliorate hyperglycemia by modulating $\beta$-cell function and insulin resistance.

Consistent with previous results, we found that the administration of SHED prevented pancreas damage and liver injury. Pancreatic islet histological analysis showed that MSC infusion prevented the destruction of pancreatic islets and produced morphologies similar to normal islets, which were organized with insulin-producing cells located centrally and glucagon-producing cells located peripherally. Nevertheless, SHED infusion ameliorated the ratio of insulin producing $\beta$-cells to total pancreatic islet cells. Meanwhile, the SHED injection significantly improved the glycogen storage in T2DM rat livers.

Mesenchymal stem cells may contribute to tissue recovery due to their immunomodulatory potential and paracrine mechanisms [15-17]. We suspect that this function may be related to SHED engraftment in the liver promoting glycogen synthesis, inhibition of glycolysis, increased gluconeogenesis and insulin resistance. In the present study, the immunohistochemical data confirmed the engraftment of a few SHED injected through the tail vein into the liver at 8 weeks after injection. Although the exact mechanism involved in stem cell homing to sites of injury remains elusive, hypoxia, inflammation and high glucose, all of which are present in diabetic livers, may induce the migration and proliferation of MSCs. In our experiments, no MSCs were found in islets with IHF, while MSCs were found in liver. We suspect that for the larger blood flow to the liver, MSCs injected through the tail vein are more likely to reach the liver instead of pancreatic islets. Another reason may be the chemotaxis of impaired liver and islets was different.

There is a decrease in glycogen synthesis, inhibition of glycolysis, increased gluconeogenesis and insulin resistance in the livers of T2DM rats. MSCs administration selectively acts on different key enzymes that play important roles. PAS staining showed that SHED administration significantly improved the glycogen storage in GK rat livers. Additionally, real-time PCR analysis showed that SHED and BMSCs significantly reversed the diabetic-induced increase of G-6-Pase and Pck1. In contrast, MSC injection significantly reversed the diabetic-induced decrease of GSK3b, GLUT2, PFKL and PK. In addition, western blotting demonstrated that SHED and BMSCs significantly reversed the diabetic-induced increase of G-6-Pase; in contrast, MSC injection significantly reversed the diabetic-induced decrease of GSK3 $\beta$, GLUT2 and PFKM. GSK3 is one of the most important regulators of glycogen synthase, which can be phosphorylated to be inactivated.
PFK is a key enzyme in the first step of catalyzing glycolysis, which is irreversible. PK catalyzes the conversion of phosphoenolpyruvate to acetone, as the last step in the process. GLUT2 is a glucose transporter expressed on the liver and islet cell membranes, and abnormal GLUT2 synthesis can lead to insulin resistance. G-6-Pase reversibly catalyzes the production of fructose-6-phosphate by glucose-6-phosphate, and PCK catalyzes the production of phosphoenolpyruvate by oxaloacetate.

\section{Conclusion}

Stem cells from human exfoliated deciduous teeth offer a potentially effective therapeutic modality for ameliorating T2DM, including hyperglycemia, insulin resistance, pancreatic islet and liver damage, and decreased glycogen synthesis, inhibition of glycolysis and increased gluconeogenesis in the liver.

\section{Abbreviations}

DM: diabetes mellitus; T2DM: type 2 diabetes mellitus; SHED: stem cells from human exfoliated deciduous teeth; MSCs: mesenchymal stem cells; GK rats: Goto-Kakizaki rats; BMSCs: bone marrow-derived mesenchymal stem cells; IPITTs: intraperitoneal insulin tolerance tests; IRTs: insulin release tests; HE: hematoxylin-eosin; PAS: periodic acid-Schiff; IHC: immunohistochemistry; PBS: phosphate buffer saline; a-MEM: a-modified Eagle's medium; FBS: fetal bovine serum; MCM: mesenchymal cells condition medium; GAPDH: glyceraldehyde3phosphate dehydrogenase; FBG: fasting blood glucose; FINS: fasting serum insulin; HOMA: homeostatic model assessment; USB-SCs: umbilical cord blood-derived mesenchymal stem cells; ADMSCs: adipose-derived mesenchymal stem cells.

\section{Authors' contributions}

NR and XW designed the experiments, analyzed the data and wrote the paper with the assistance of $L G$ and $Y Z$. NR, XW, JX, JL performed the experiments.

$Y Z$ revised and checked the language. All authors read and approved the final manuscript.

\section{Author details}

1 Department of Pediatric Dentistry, Peking University School and Hospital of Stomatology, 22 Zhongguancun Avenue South, Haidian District, Beijing 100081, People's Republic of China. ${ }^{2}$ Department of Oral Emergency Department, Peking University School and Hospital of Stomatology, 22 Zhongguancun Avenue South, Haidian District, Beijing 100081, People's Republic of China. ${ }^{3}$ Department of Stomatology, Shenzhen Children's Hospital, No. 7019, Yitian Road, Shenzhen 518026, People's Republic of China.

\section{Acknowledgements \\ Not applicable.}

Competing interests

The authors declare that they have nocompeting interests.

\section{Availability of data and materials}

All data generated and/or analyzed during this study are available from the corresponding author upon reasonable request.

Consent for publication

Not applicable. 


\section{Ethics approval and consent to participate}

SHED were isolated from surgical waste of individual. Sample were obtained after written informed consent from donors and their parents. All animal experiments were conducted in accordance with the protocols and guidelines of the PU IRB laboratory Animal Welfare Committee (No. LA2018231).

\section{Funding}

The research was supported by the National Natural Science Foundation of China (303076129) and the Peking University Taisheng Stomatology Development Fund (No. 94000-6672-H78).

\section{Publisher's Note}

Springer Nature remains neutral with regard to jurisdictional claims in published maps and institutional affiliations.

Received: 3 December 2018 Accepted: 19 February 2019 Published online: 28 February 2019

\section{References}

1. World Health Organization. Global report on diabetes; 2006. http://www. who.int/diabetes/global-report/en/.

2. Association AD. Diagnosis and classification of diabetes mellitus. Diabetes Care. 2012;31(Suppl. 1):S55-60.

3. Inzucchi SE. Oral antihyperglycemic therapy for type 2 diabetes: scientific review. JAMA. 2002;287(3):373.

4. Rangel EB. The metabolic and toxicological considerations for immunosuppressive drugs used during pancreas transplantation. Expert Opin Drug Metab Toxicol. 2012;8(12):1531-48

5. Kandaswamy R, Skeans MA, Gustafson SK, et al. OPTN/SRTR 2013 annual data report: pancreas. Am J Transplant. 2015;15(S2):1-20.

6. Sharpe PT. Dental mesenchymal stem cells. Development. 2016;143(13):2273-80.

7. Shin TH, Kim HS, Choi SW, Kang KS. Mesenchymal stem cell therapy for inflammatory skin diseases: clinical potential and mode of action. Int J Mol Sci. 2017;18(2):E244.

8. Horwitz EM, Prockop DJ, Fitzpatrick LA, Koo WW, Gordon PL, Neel M, Sussman M, Orchard P, Marx JC, Pyeritz RE, Brenner MK. Transplantability and therapeutic effects of bone marrow-derived mesenchymal cells in children with osteogenesis imperfecta. Nat Med. 1999;5:309-13.

9. Haber Tom, Baruch Limor, Machlufa Marcelle. Ultrasound-mediated mesenchymal stem cells transfection as a targeted cancer therapy platform. Sci Rep. 2017;7:42046

10. Boumaza I, Srinivasan S, Witt WT, et al. Autologous bone marrow derived rat mesenchymal stem cells promote PDX-1 and insulin expression in the islets, alter $T$ cell cytokine pattern and preserve regulatory $T$ cells in the periphery and induce sustained normoglycemia. J Autoimmun. 2009;32:33-42.

11. Jurewicz M, Yang S, Augello A, Godwin JG, Moore RF, Azzi J, Fiorina P, Atkinson M, Sayegh MH, Abdi R. Congenic mesenchymal stem cell therapy reverses hyperglycemia in experimental type 1 diabetes. Diabetes. 2010:59:3139-47.

12. Ezquer F, Ezquer M, Simon V, Conget $P$. The antidiabetic effect of MSCs is not impaired by insulin prophylaxis and is not improved by a second dose of cells. PLoS ONE. 2011;6:e16566.

13. Si Y, Zhao Y, Hao H, Liu J, Guo Y, Mu Y, Shen J, Cheng Y, Fu X, Han W. Infusion of mesenchymal stem cells ameliorates hyperglycemia in type 2 diabetic rats identification of a novel role in improving insulin sensitivity. Diabetes. 2012;61(6):1616-25.

14. Bhansali A, Upreti V, Khandelwal N, Marwaha N, Gupta V, Sachdeva N, Sharma RR, Saluja K, Dutta P, Walia R, Minz R, Bhadada S, Das S,
Ramakrishnan S. Efficacy of autologous bone marrow-derived stem cell transplantation in patients with type 2 diabetes mellitus. Stem Cells Dev. 2009:18(10):1407-16.

15. Fiorina P, Jurewicz M, Augello A, Vergani A, Dada S, La Rosa S, Selig M, Godwin J, Law K, Placidi C, Smith RN, Capella C, Rodig S, Adra CN, Atkinson M, Sayegh MH, Abdi R. Immunomodulatory function of bone marrow-derived mesenchymal stem cells in experimental autoimmune type 1 diabetes. J Immunol. 2009;183:993-1004.

16. Ben-Ami E, Berrih-Aknin S, Miller A. Mesenchymal stem cells as an immunomodulatory therapeutic strategy for autoimmune diseases. Autoimmun Rev. 2011;10:410-5.

17. Lechner A, Yang YG, Blacken RA, Wang L, Nolan AL, Habener JF. No evidence for significant transdifferentiation of bone marrow into pancreatic beta-cells in vivo. Diabetes. 2004:53(3):616-23.

18. Maccario R, Podestà M, Moretta A, Cometa A, Comoli P, Montagna D, Daudt L, Ibatici A, Piaggio G, Pozzi S, Frassoni F, Locatelli F. Interaction of human mesenchymal stem cells with cells involved in alloantigenspecific immune response favors the differentiation of CD4p T-cell subsets expressing a regulatory/suppressive phenotype. Haematologica. 2005:90:516-25.

19. Li N, Hua J. Interactions between mesenchymal stem cells and the immune system. Cell Mol Life Sci. 2017;74(13):2345-60.

20. Rasmusson I, Ringden O, Sundberg B, et al. Mesenchymal stem cells inhibit lymphocyte proliferation by mitogens and alloantigens by different mechanisms. Exp Cell Res. 2005;305:33-41.

21. Phinney DG, Prockop DJ. Mesenchymal stromal cells and immunomodulation: a gathering of regulatory immune cells. Cytotherapy. 2016;18(2):160-71.

22. Caplan Al, Dennis JE. Mesenchymal stem cells as trophic mediators. J Cell Biochem. 2006;98:1076-84.

23. Miura M, Gronthos S, Zhao M, Lu B, Fisher LW, Robey PG, Shi S. SHED: stem cells from human exfoliated deciduous teeth. Proc Natl Acad Sci USA. 2003;100(10):5807-12.

24. Shimojima C, Takeuchi H, Jin S, Parajuli B, Hattori H, Suzumura A, Hibi $H$, Ueda M, Yamamoto A. Conditioned medium from the stem cells of human exfoliated deciduous teeth ameliorates experimental autoimmune encephalomyelitis. J Immunol. 2016;196(10):4164-71.

25. Kanafi MM, Rajeshwari YB, Gupta S, Dadheech N, Nair PD, Gupta PK, Bhonde RR. Transplantation of islet-like cell clusters derived from human dental pulp stem cells restores normoglycemia in diabetic mice. Cytotherapy. 2013;15(10):1228-36.

26. Yamaza T, Alatas FS, Yuniartha R, Yamaza H, Fujiyoshi JK, Yanagi Y, Yoshimaru K, Hayashida M, Matsuura T, Aijima R, Ihara K, Ohga S, Shi S, Nonaka $K$, Taguchi T. In vivo hepatogenic capacity and therapeutic potential of stem cells from human exfoliated deciduous teeth in liver fibrosis in mice. Stem Cell Res Ther. 2015;6:171.

27. Yamaza T, Kentaro A, Chen C, Liu Y, Shi Y, Gronthos S, Wang S, Shi S. Immunomodulatory properties of stem cells from human exfoliated deciduous teeth. Stem Cell Res Ther. 2010;1(1):5.

28. Taghipour Z, Karbalaie K, Kiani A, Niapour A, Bahramian H, Nasr-Esfahani $\mathrm{MH}$, Baharvand $\mathrm{H}$. Transplantation of undifferentiated and induced human exfoliated deciduous teeth-derived stem cells promote functional recovery of rat spinal cord contusion injury model. Stem Cells Dev. 2012;21(10):1794-802.

29. Kuwabara WMT, Panveloski-Costa AC, Yokota CNF, Pereira JNB, Filho JM, Torres RP, Hirabara SM, Curi R, Alba-Loureiro TC. Comparison of Goto-Kakizaki rats and high fat diet-induced obese rats: are they reliable models to study type 2 diabetes mellitus? PLoS ONE. 2017;12(12):e0189622.

30. Portha B, Lacraz G, Dolz M, Giroix MH, Homo-Delarche F, Movassat J. Issues surrounding beta-cells and their roles in type 2 diabetes. What tell us the GK rat model. Expert Rev Endocrinol Metab. 2007;2:785-95. 